\title{
Project SUCCESS: Results from a Randomized Controlled Trial
}

\author{
Karen S. Calabro, ${ }^{1}$ Salma Marani, ${ }^{1}$ Tracey Yost, ${ }^{2}$ Janice Segura, ${ }^{1}$ Mary Mullin Jones, ${ }^{1}$ \\ Sheryl Nelson, ${ }^{1}$ Carl de Moor, ${ }^{3}$ and Alexander V. Prokhorov ${ }^{1}$ \\ ${ }^{1}$ Department of Behavioral Science, Division of Cancer Prevention and Population Sciences, The University of Texas MD Anderson \\ Cancer Center, 1515 Holcombe Boulevard, Unit 1330, Houston, TX 77030, USA \\ ${ }^{2}$ Department of Health Disparities Research, Division of Cancer Prevention and Population Sciences, The University of Texas MD \\ Anderson Cancer Center, 1400 Pressler St., Houston, TX 77030, USA \\ ${ }^{3}$ Registrat-MAPI, 2343 Alexandria Drive, Suite 100, Lexington, KY 40504-3276, USA
}

Correspondence should be addressed to Alexander V. Prokhorov, aprokhor@mdanderson.org

Received 30 April 2012; Accepted 30 May 2012

Academic Editors: C. Banwell and C. Castro

Copyright ( 2012 Karen S. Calabro et al. This is an open access article distributed under the Creative Commons Attribution License, which permits unrestricted use, distribution, and reproduction in any medium, provided the original work is properly cited.

\begin{abstract}
The present investigation tested whether an enhanced smoking cessation program produced higher cessation rates for participants than for controls. Participants in the enhanced intervention condition received in-person motivational counseling with health feedback, a tailored internet-based program, and nicotine patch. Participants in the control group received a smoking cessation self-help manual and nicotine patch. This randomized controlled trial was conducted at a 4-year university with a student body of 32,000 . Five hundred-nine students who smoked $\geq 1$ cigarette daily were individually randomized into the enhanced intervention and control groups. Over a 3-month period, participants in the enhanced intervention condition attended two personal sessions with smoking cessation counselors. Participants in both conditions were reassessed for smoking status 12 months post-baseline. Multivariate logistic regression techniques were used to analyze the data using the intent to treat approach. Results indicated that the odds of smoking cessation were 2 times larger for the enhanced intervention group than controls (odds ratio $=2.3,95 \%$, confidence interval $=1.3,3.9, P<.01$ ). This study begins to fill research gaps regarding college students and smoking cessation. Suggestions for future advancements in smoking cessation interventions for college students are provided.
\end{abstract}

\section{Introduction}

Tobacco-related disease in the United States is attributed to 443,000 deaths annually. Annual economic losses associated with smoking exceed $\$ 193$ billion [1]. Thirty-eight hundred adolescents experiment with cigarette smoking each day [2]. Estimates indicate that 1 in 5 may gradually become established adult smokers [3]. Evidence from case control studies indicated that the majority of tobacco-related attributable risk of disease could be prevented if adult smokers quit before age 30 [4]. Tobacco cessation efforts for young adults have not been addressed as extensively as for adolescents and more mature adults [5]. There are two levels of daily cigarette use among those in the 18-24-year-old age segment. For those enrolled in college, the daily prevalence for cigarette use is $7.6 \%$ and for those entering the workforce full time the prevalence is $14.8 \%$ [6]. Young adults attending college are faced with new possibilities and academic pressures [7]. Unhealthy behaviors such as smoking and heavy alcohol use can be initiated and increased [8]. Because colleges are organized locations with concentrated young adult populations, campuses offer opportunities for increasing the reach of evidence-based smoking cessation interventions.

Clinical approaches have been used to assist student smokers to quit smoking in colleges and universities [5]. A review of previous studies yielded investigations with short follow-up periods and potentially too few participants for powering evaluations [7, 9-11]. Modest results on smoking cessation outcomes among college students have been achieved. A campus-based randomized controlled trial using an on-line college student magazine that incorporated tobacco cessation advice was reported. From a high engagement perspective, participants were adherent in logging into the study website as directed for 20 sessions [12]. An 
inclusion criterion for joining the study was that one had to smoke $\geq 1$ cigarette in a month, allowing occasional smokers to be study eligible, a group that is challenging to recruit. Participants in the intervention group were more likely to report abstinence for a 30-day period than controls. Quitting for 30 days among those who smoke one cigarette a month may not be as difficult as for those with daily consumption [13]. Further research impact was decreased because of the potential for contamination since intervention participants may have simultaneously joined a separate smoking cessation cash prize competition [12]. Improvements are needed to produce successful interventions and facilitate the delivery of smoking cessation services to young adults [14].

The primary aim for Project SUCCESS involved assessing whether the smoking cessation program that combined in-person motivational counseling with health feedback, a tailored internet-based program, and nicotine patch in the enhanced intervention (EI) increased rates of smoking cessation greater than standard-care (SC), which consisted of a smoking cessation self-help manual and nicotine patch at 12-month followup.

\section{Methods}

\subsection{Study Design}

2.1.1. Participants and Procedures. College students were recruited at a state funded university that was not a tobaccofree campus. Over 32,000 undergraduate and graduate students attended the predominately commuter university located in the southwestern United States. This study took place on the campus setting and the internet (with a technology component). The MD Anderson Cancer Center Institutional Review Board approved the investigation protocol. Project SUCCESS was advertised through flyers in campus halls, newsletters, e-mail, and during presentations in classes. Outreach recruitment strategies by study personnel involved appearing at residence halls, school registration areas, and social functions. Smoking cessation counselors contacted interested participants by telephone and e-mail messaging to screen candidates for eligibility. Inclusion criteria included being aged 18 to 35 years, enrolled students, a current smoker who smoked $\geq 1$ cigarette per day for at least 6 months, being able to read English, and having access to internet and telephone. Exclusion criteria included those who had a close friend or relative in the study, using tobacco products other than cigarettes, those enrolled in other smoking cessation programs, those who could not use nicotine replacement therapy, those using other quitting aid therapies not supplied by the study, and those who were dependent on recreational drugs. Informed consent was obtained. The simple randomization sequence was generated by a statistical software package. Participants were recruited from February 1, 2005 to July 12, 2006. Participants were modestly incentivized with $\$ 10$ campus bookstore gift cards distributed after engaging in individual study activities. At baseline participants from both study groups met with their assigned counselors and salivary cotinine testing was performed to confirm smoking status (NicAlert saliva testing kit) [15]. At baseline, all study participants were required to test positive for cotinine. Nicotine replacement therapy was offered to participants if students smoked $\geq 5$ cigarettes daily. At 3-months and 12-months after recruitment counselors met with participants.

2.1.2. Group Condition: Standard Care. For this study, we used the term "standard care" in reference to the control group. Standard care (SC) participants completed questionnaires assessing demographics and standard tobacco-related concerns at the first session. Participants received a smoking cessation self-help written material from the National Cancer Institute. At two subsequent in-person visits, 1page handouts extracted from the same self-help material were distributed to SC participants. In these sessions $(\leq 5$ minutes), the counselor provided minimal counseling, and no persuasive communication or assistance to participants. If participants raised questions, the counselor answered questions and directed participants to the appropriate section in the written materials.

2.1.3. Group Condition: Enhanced Intervention. The EI group received multicomponent interventions designed to increase participants' perceptions of vulnerability to the harms of smoking and increase self-efficacy and skills for ability to quit. Smoking cessation counselors assisted participants in reevaluating smoking and setting goals for reductions in smoking behaviors. The length of sessions ranged from 30 to 60 minutes. This condition was modeled after our previous tobacco intervention research among college students [16, 17].

(1) Expert Computer Software System. This system was developed for SUCCESS, allowing systematic delivery of the intervention. The expert system handled participant scheduling, data collection, and processing. The computer screen allowed counselors to view results and facilitated consistent delivery of individual plans based on the participants' responses and stage of change for smoking cessation. Personalized newsletters were printed and distributed to inform participants about scores for their respiratory health parameters compared to nonsmokers.

(2) Theoretical Basis and Motivational Interviewing. Obtaining input from the transtheoretical model, one of the most widely used behavior change theories, we drew upon its concepts to attempt to activate an individual's progress toward smoking cessation. The transtheoretical model of health behavior change was conceptualized to explain how smokers progress toward cessation [18]. According to the stages of change component of the model, progress toward cessation is grouped into five stages: precontemplation, contemplation, preparation, action, and maintenance. The precontemplation stage is a state where a smoker does not intend to quit. The contemplation stage is consistent with a smoker considering a desire to quit or need to change but makes no actual effort. Preparation is a stage where one is 
planning to quit smoking in the next month and had made a quit attempt in the past 12 months. In action, the person successfully quits for 6 months; in maintenance sustained smoking cessation is observed.

Motivational interviewing (MI) is described as a "clientcentered, directive method for enhancing intrinsic motivation to change by exploring and resolving ambivalence." [19] The client's interests are the primary focus as the counselor's strategies help individuals move toward change [19]. These techniques were applied by counselors in the EI group during the health feedback and in-person counseling. These motivational enhancements and health feedback were designed to sensitize smokers about the negative health effects of smoking.

(3) Health Feedback. In the EI group, physical measurements, exhaled carbon monoxide, and lung function assessed by spirometry were tested and results immediately reviewed among participants. The Micro CO respiratory monitor (Micro Direct, Lewiston, ME) was used to measure the parts per million CO to air and percentage of carboxyhemoglobin. Carbon monoxide levels of eight parts per million or higher were used as the reference value to indicate current smoking [20]. Counselors tested lung function among participants with a spirometer (SpiroCard; QRS, Minneapolis, MN). Using measured forced expiratory volume at one second, the expert system computed the lung age [21]. Lung age was determined by comparing the smoker's spirometry results to referent values for the lung conditions of nonsmokers of the same age, gender and height $[22,23]$. Smokers were given feedback that their lungs were normal if the lung age was less than or equal to the chronological age. Smokers tend to score higher for lung age than chronological age. Further, counselors reviewed and conveyed the significance of respiratory symptoms (self-reported), smoking history, and presence of preexisting asthma and bronchitis.

(4) Web-Based Booster Sessions. Computer and internet access was universal on campus permitting EI participants access to the intervention website. Between sessions 1 and 2 , five computer-based booster sessions were delivered online and accessed by participants actively logging on to the SUCCESS website. In addition, participants were urged to visit the web module, to obtain assistance with coping with triggers for smoking use of the nicotine replacement therapy, and achieving abstinence. The program and website were designed to serve as convenient resources when participants encountered difficulty in achieving cessation. Resources on the internet site remained available to participants over the 12-month study period.

2.1.4. Counselor Training. In regards to smoking cessation counselor training, prior to participant recruitment, the two EI counselors attended a 2-day motivational interviewing workshop at the Baylor College of Medicine. The counselor training was designed to teach and enhance skills for counselors for encouraging smokers to achieve behavior changes. Additionally, counselors were trained to provide personalized feedback about the adverse consequences of smoking. Proper conduct of spirometry was taught with instructions from a course approved by the National Institute for Occupational Safety and Health. Also, counselors were trained to measure both salivary cotinine and carbon monoxide. Counselors practiced with volunteers and received feedback before working with study participants.

2.2. Measures. The baseline survey asked about participant demographics. We report on those participant characteristics used in the analysis of outcomes (see Table 1). The Center for Epidemiologic Studies Depression (CES-D) scale was administered to screen for depressive symptoms [24]. The time frame for report of the depressive symptoms was past week. Using standard scales from smoking cessation studies, the survey items captured the following: number of years smoked cigarettes, cigarettes smoked per day, decisions for smoking [25], self-efficacy/temptation, processes of change [26], quitting history, methods used to try to quit, reasons for relapse, tobacco craving, withdrawal symptoms [27], and temptations to smoke [28]. Using an American Thoracic Society standard questionnaire, participants were asked about frequency of cough, phlegm, shortness of breath, chest pain, and fatigue [23]. Follow-up surveys asked about tobacco use and abstinence, methods used for quitting, completed the Fagerstrom Test for Nicotine Dependence (FTND) [29], respiratory symptoms assessment [23], and reasons for relapses.

\section{Results}

3.1. Baseline Characteristics. A total of 509 participants were recruited. For the overall sample, the mean age was $23.8 \pm$ 4.0 years (see Table 2 ). Approximately half of participants were male (52.7\%). The majority were Caucasian (70.5\%). For alcohol use, about $18 \%$ reported their alcohol consumption as heavy or frequent. The majority consumed alcohol moderately or rarely with only about $9 \%$ abstaining from alcohol. The assessment of depressive symptomatology indicated that half scored high for depressive symptoms. For smoking-related characteristics, the majority of participants indicated that they were seriously thinking of quitting in the next 30 days (i.e., the preparation stage of change for smoking cessation). Forty-one percent were thinking about quitting within the next 6 months (contemplation). Six percent reported that they were not seriously thinking about quitting smoking (precontemplation). Eleven percent reported becoming regular daily smokers in the past 6 to 12 months. The majority had been smoking 5 years or more. Fifty-four percent smoked half a pack or less daily. In regards to nicotine dependence (FTND), over twothirds reported low to very low nicotine dependence. The data about the frequency of respiratory symptoms were reported previously [30]. With one exception (the EI group was comprised of slightly more smokers consuming 6 to 10 cigarettes regularly), results indicated the groups were balanced statistically. 
TABLE 1: Key measures used in outcome analysis.

\begin{tabular}{|c|c|c|c|c|c|}
\hline Construct & Scale & Items & Response categories & Scoring & $\begin{array}{c}\text { Cronbach } \\
\text { alpha }\end{array}$ \\
\hline $\begin{array}{l}\text { Readiness to quit } \\
\text { smoking }\end{array}$ & $\begin{array}{l}\text { Stages of change: } \\
\text { (1) thinking about } \\
\text { quitting, (2) number of } \\
\text { quit attempts. }\end{array}$ & 2 & $\begin{array}{l}\text { Yes, within the next six months; } \\
\text { and no, not thinking of quitting. } \\
\text { The responses were none, one } \\
\text { time, two times, three to five } \\
\text { times, and six or more times. }\end{array}$ & $\begin{array}{l}\text { Scoring } 1-3 \text {. Report in next } 6 \\
\text { months, in contemplation stage. } \\
\text { Not thinking about quitting is } \\
\text { contemplation stage. If trying to } \\
\text { quit, they are in the action stage. }\end{array}$ & $\mathrm{n} / \mathrm{a}$ \\
\hline $\begin{array}{l}\text { Nicotine } \\
\text { dependence }\end{array}$ & $\begin{array}{l}\text { Fagerström Test for } \\
\text { Nicotine Dependence } \\
\text { (FTND). }\end{array}$ & 6 & $\begin{array}{l}\text { Multiple choice and } \\
\text { dichotomous }\end{array}$ & $\begin{array}{l}\text { Scores: 0-2/very low; 3-4/low, } \\
\text { 5/medium, 6-7/high, 8-10/very } \\
\text { high. }\end{array}$ & .61 \\
\hline $\begin{array}{l}\text { Depressive } \\
\text { symptoms }\end{array}$ & $\begin{array}{l}\text { Center for } \\
\text { Epidemiologic Studies } \\
\text { of Depressive Symptoms } \\
\text { Scale (CES-D). } \\
\text { Used to measure severity } \\
\text { of depression in general } \\
\text { population. }\end{array}$ & 20 & $\begin{array}{l}1 \text { (rarely) to } 4 \text { (most all of the } \\
\text { time). Inquiring about the } \\
\text { degree of occurrence during the } \\
\text { last week. }\end{array}$ & $\begin{array}{l}\text { Cut-off scores } \\
\text { Scores }<16 \text { categorized participants } \\
\text { as not at high risk for depressive } \\
\text { symptoms. } \\
\text { Scores }>16 \text { indicated that the } \\
\text { participants were reporting high } \\
\text { levels of depressive symptoms. }\end{array}$ & .78 \\
\hline Alcohol use & $\begin{array}{l}\text { Do you currently drink } \\
\text { alcoholic beverages? }\end{array}$ & 1 & $\begin{array}{l}\text { Four responses: (1) yes, heavily } \\
\text { and/or frequently, (2) yes, } \\
\text { moderately and/or rarely, (3) no, } \\
\text { (4) I would rather not answer. }\end{array}$ & Scoring 1-4. & $\mathrm{n} / \mathrm{a}$ \\
\hline
\end{tabular}

\subsection{Noncompleters}

3.2.1. Completers versus Noncompleters at 12-Month Followup. At 12-month followup, the study retention rate was 46\% (236 of 509 enrolled). We compared completers and noncompleters on baseline demographic characteristics, smokingrelated variables, depression and alcohol and drug use. Females were significantly more likely to complete the study than males, $\chi^{2}(1, N=509)=5.6, P=.02$. Hispanics were more likely to be noncompleters compared to nonHispanics, $\chi^{2}(1, N=509)=1.2, P=.03$. No differences between noncompleters and completers were found for the following: use of alcohol, depressive symptoms, and other smoking-related characteristics.

3.2.2. Baseline Group Differences by Condition. A total of 236 students completed the 12-month assessment with 158 in the EI group and 78 in the SC group. As followed from baseline, with the exception of there being slightly heavier smokers in the EI group, no baseline group differences were found in the subsample that completed the final survey for demographic characteristics, smoking-related variables, or alcohol use (Table 3).

\subsubsection{Abstinence at 12-Month Followup and Biochemical} Validation. We preferred that quitters produced a salivary cotinine value $\leq 5 \mathrm{ng} / \mathrm{mL}$ to validate nonsmoking status. Forty-six of $79(58 \%)$ at the 12-month assessment who reported abstinence produced cotinine samples; $100 \%$ of these samples were negative for cotinine. Challenges in meeting with participants at the final assessment to obtain salivary samples compelled waiving biochemical validation for those who could not provide samples. We decided to use self-reported abstinence in the final analysis. The proportion of participants who were abstinent at 12-month followup was compared between EI and SC groups. Logistic regression models were used to test for an overall treatment group difference. Abstinence (yes versus no) was regressed onto treatment group variable (EI versus SC). Selected covariates for the final model included age, gender, ethnicity, baseline FTND, frequency of alcohol use, and presence of depressive symptoms. The regression coefficient associated with the treatment group variable in this analysis represents the overall $\log$ odds of being abstinent in the EI group relative to the SC group at 12-month followup controlling for the covariates. To test for an overall treatment group difference, the likelihood ratio test was used to examine whether the difference between the EI and SC groups is statistically and numerically significant. Of the 509 students recruited at baseline, 236 completed the final assessment, 158 in EI and 78 in SC. At 12-month followup, 79 (33.5\%) of the final sample quit smoking. There were a total of 153 noncompleters in SC (66\%) and 120 in EI (43\%) (Figure 1). Noncompleters did not differ between EI and SC for demographic variables, smoking-related variables, and presence of depressive symptoms or frequency of alcohol intake. Twenty percent of participants were quitters in the EI group compared to $10 \%$ in the SC group, $(P<.01)$ (odds ratio $=2.3,95 \%$, confidence interval $=1.3,3.9, P<.01)$.

\section{Discussion}

The present study reports on the outcomes of Project SUCCESS. This was a randomized controlled trial testing the cessation rates achieved for a smoking cessation program. In this study, the intervention consisted of combined inperson motivational counseling with health feedback, and a tailored internet-based program. The cessation rates for the intervention were compared to those for the controls. The odds of smoking cessation were 2 times larger for the 
TABLE 2: University campus-based smoking cessation intervention-participants at baseline.

\begin{tabular}{|c|c|c|c|c|c|c|c|}
\hline \multirow{4}{*}{$\begin{array}{l}\text { Study group } \\
\text { Variable }\end{array}$} & \multicolumn{7}{|c|}{ Baseline } \\
\hline & \multicolumn{2}{|c|}{ Total ${ }^{*}$} & \multicolumn{2}{|c|}{$\mathrm{SC}^{1}$} & \multicolumn{2}{|c|}{$\mathrm{EI}^{2}$} & \multirow{3}{*}{$P$ value ${ }^{3}$} \\
\hline & $n$ & $\%$ & $n$ & $\%$ & $n$ & $\%$ & \\
\hline & 509 & 231 & 45.3 & 278 & 54.6 & & \\
\hline \multicolumn{8}{|l|}{ Demographics } \\
\hline Age (mean, SD) & $23.8(4.0)$ & & $24.0(4.3)$ & & $23.6(3.8)$ & & .2 \\
\hline Gender & & & & & & & .7 \\
\hline Female & 241 & 47.3 & 107 & 46.3 & 134 & 48.2 & \\
\hline Male & 268 & 52.7 & 124 & 53.7 & 144 & 51.8 & \\
\hline Ethnicity & & & & & & & .7 \\
\hline Spanish/Hispanic & 56 & 11.0 & 27 & 11.0 & 29 & 10.4 & \\
\hline Not Spanish/Hispanic & 452 & 89.0 & 204 & 89.0 & 248 & 89.2 & \\
\hline Racial background & & & & & & & .7 \\
\hline Asian & 106 & 20.8 & 54 & 23.4 & 52 & 18.8 & \\
\hline Black & 32 & 6.3 & 15 & 6.5 & 17 & 6.1 & \\
\hline White & 359 & 70.5 & 157 & 68.0 & 202 & 72.7 & \\
\hline Other & 12 & 2.3 & 5 & 3.2 & 6 & 2.1 & \\
\hline Alcohol consumption & & & & & & & .4 \\
\hline Heavily and or frequently & 91 & 17.9 & 36 & 15.6 & 55 & 19.8 & \\
\hline Moderately or rarely & 362 & 71.1 & 167 & 72.3 & 195 & 70.1 & \\
\hline None & 47 & 9.2 & 25 & 10.8 & 22 & 7.9 & \\
\hline Rather not answer & 9 & 1.8 & 3 & 1.3 & 6 & 2.2 & \\
\hline Depressive symptoms (CES-D) & & & & & & & .4 \\
\hline Low & 255 & 50.1 & 111 & 48.0 & 144 & 51.8 & \\
\hline High & 254 & 49.9 & 120 & 51.9 & 134 & 48.2 & \\
\hline \multicolumn{8}{|l|}{ Smoking-related characteristics } \\
\hline Stage of change for cessation & & & & & & & .8 \\
\hline Preparation & 268 & 52.6 & 125 & 54.1 & 143 & 51.4 & \\
\hline Contemplation & 210 & 41.2 & 92 & 39.8 & 118 & 42.4 & \\
\hline Precontemplation & 31 & 6.1 & 14 & 6.1 & 17 & 6.1 & \\
\hline Nicotine dependence (FTND) & & & & & & & .6 \\
\hline Very low & 208 & 41.1 & 90 & 39.3 & 118 & 42.6 & \\
\hline Low & 140 & 27.7 & 59 & 25.8 & 81 & 29.2 & \\
\hline Medium & 50 & 9.9 & 25 & 10.9 & 25 & 9.0 & \\
\hline High & 69 & 13.6 & 34 & 14.8 & 35 & 12.6 & \\
\hline Very high & 39 & 7.7 & 21 & 9.2 & 18 & 6.5 & \\
\hline Cigarettes per day & & & & & & & .04 \\
\hline $1-5$ & 115 & 22.6 & 53 & 22.9 & 62 & 22.3 & \\
\hline $6-10$ & 158 & 31.0 & 59 & 25.5 & 99 & 35.6 & \\
\hline $11-15$ & 91 & 17.9 & 45 & 19.5 & 46 & 16.5 & \\
\hline $16-20$ & 97 & 19.1 & 46 & 19.9 & 51 & 18.3 & \\
\hline $21-30$ & 40 & 7.9 & 21 & 9.1 & 19 & 6.8 & \\
\hline $31+$ & 8 & 1.6 & 7 & 3.0 & 1 & .4 & \\
\hline History of cigarette smoking & & & & & & & .6 \\
\hline Less than 6 mo & 14 & 2.8 & 4 & 1.7 & 10 & 3.6 & \\
\hline 6-12 mo & 43 & 8.4 & 19 & 8.2 & 24 & 8.6 & \\
\hline $1-5$ years & 188 & 36.9 & 84 & 36.4 & 104 & 37.4 & \\
\hline 5 years or greater & 264 & 51.9 & 124 & 53.7 & 140 & 50.4 & \\
\hline
\end{tabular}

* "Total" pertains to the total sample. ${ }^{1}$ SC: standard Care. ${ }^{2} \mathrm{EI}$ : enhanced intervention. ${ }^{3} \mathrm{P}$ value reflects significance of comparisons between conditions (SC versus EI). Percentages for each variable may not add up to 100 because of missing data. 
TABLE 3: University campus-based smoking cessation intervention at 12-month assessment.

\begin{tabular}{|c|c|c|c|c|c|c|c|c|c|c|c|c|c|c|}
\hline \multirow{4}{*}{$\begin{array}{l}\text { Study group } \\
\text { Variable }\end{array}$} & \multicolumn{7}{|c|}{ Baseline } & \multicolumn{7}{|c|}{ 12-month assessment } \\
\hline & \multicolumn{2}{|c|}{ Total* } & \multicolumn{2}{|c|}{$\mathrm{SC}^{1}$} & \multicolumn{2}{|c|}{$\mathrm{EI}^{2}$} & & \multicolumn{2}{|c|}{ Total } & \multicolumn{2}{|c|}{ SC } & \multicolumn{2}{|l|}{ EI } & \multirow{3}{*}{$P$ value $^{3}$} \\
\hline & $n$ & $\%$ & $n$ & $\%$ & $n$ & $\%$ & $P$ value ${ }^{3}$ & $n$ & $\%$ & $n$ & $\%$ & $n$ & $\%$ & \\
\hline & 236 & 44.0 & 78 & 33.0 & 158 & 66.0 & & 236 & & 78 & & 158 & & \\
\hline \multicolumn{15}{|l|}{ Demographics } \\
\hline Age (mean, SD) & $24.5(.3)$ & & $24.9(.5$ & & $24.3(.3)$ & & .3 & $24.5(.3)$ & & $24.9(.5)$ & & $24.3(.3)$ & & .3 \\
\hline Gender & & & & & & & .5 & & & & & & & .45 \\
\hline Female & 125 & 53.0 & 44 & 56.4 & 81 & 51.3 & & 125 & 53.0 & 44 & 56.4 & 81 & 51.3 & \\
\hline Male & 111 & 47.0 & 34 & 43.6 & 77 & 48.7 & & 111 & 47.0 & 34 & 43.6 & 77 & 48.7 & \\
\hline Ethnicity & & & & & & & .9 & & & & & & & .9 \\
\hline Spanish/Hispanic & 22 & 9.4 & 7 & 9.0 & 15 & 9.5 & & 22 & 9.4 & 7 & 8.9 & 15 & 9.5 & \\
\hline Not Spanish/Hispanic & 213 & 90.6 & 71 & 91.0 & 142 & 90.4 & & 213 & 90.6 & 71 & 91.1 & 142 & 90.4 & \\
\hline Racial background & & & & & & & .4 & & & & & & & .4 \\
\hline Asian & 44 & 18.7 & 19 & 24.4 & 25 & 15.9 & & 44 & 18.7 & 19 & 24.4 & 25 & 15.9 & \\
\hline Black & 14 & 6.0 & 3 & 3.8 & 11 & 7.0 & & 14 & 6.0 & 3 & 3.8 & 11 & 7.0 & \\
\hline White & 172 & 73.2 & 54 & 69.2 & 118 & 75.2 & & 172 & 73.2 & 54 & 69.2 & 118 & 75.2 & \\
\hline Other & 5 & 2.1 & 2 & 2.6 & 3 & 1.9 & & 5 & 2.2 & 2 & 1.7 & 3 & 1.9 & \\
\hline Alcohol consumption & & & & & & & 6 & & & & & & & .9 \\
\hline Heavily and or frequently & 41 & 17.4 & 11 & 14.1 & 30 & 19.0 & & 27 & 11.4 & 9 & 11.5 & 18 & 11.4 & \\
\hline Moderately or rarely & 166 & 70.3 & 57 & 73.1 & 109 & 69.0 & & 160 & 67.8 & 54 & 69.2 & 106 & 67.1 & \\
\hline None & 23 & 9.7 & 7 & 9.0 & 16 & 10.1 & & 47 & 19.9 & 15 & 19.2 & 32 & 20.2 & \\
\hline Rather not answer & 6 & 2.5 & 3 & 3.8 & 3 & 1.9 & & 2 & .8 & 0 & 0 & 2 & 1.3 & \\
\hline Depressive symptoms (CES-D) & & & & & & & 6 & & & & & & & \\
\hline Low & 119 & 50.4 & 41 & 52.6 & 78 & 49.4 & & - & - & - & - & - & - & - \\
\hline High & 117 & 49.6 & 37 & 47.4 & 80 & 50.6 & & & & & & & & \\
\hline \multicolumn{15}{|l|}{ Smoking-related characteristics } \\
\hline Stage of change for cessation & & & & & & & 1.0 & & & & & & & \\
\hline Preparation & 127 & 53.8 & 42 & 53.8 & 85 & 53.8 & & & & & & & & \\
\hline Contemplation & 94 & 39.8 & 31 & 39.7 & 63 & 39.9 & & - & - & - & - & - & - & - \\
\hline Precontemplation & 15 & 6.4 & 5 & 6.4 & 10 & 6.3 & & & & & & & & \\
\hline Nicotine dependence & & & & & & & .5 & & & & & & & .13 \\
\hline Very low & 97 & 41.1 & 30 & 39.0 & 67 & 42.7 & & 180 & 76.3 & 52 & 67.0 & 128 & 81.0 & \\
\hline Low & 67 & 28.6 & 19 & 24.7 & 48 & 30.6 & & 35 & 14.8 & 16 & 21.5 & 19 & 12.0 & \\
\hline Medium & 19 & 8.1 & 8 & 10.4 & 11 & 7.0 & & 12 & 5.1 & 6 & 7.6 & 6 & 3.8 & \\
\hline High & 34 & 14.5 & 12 & 15.6 & 22 & 14.0 & & 7 & 3.0 & 3 & 3.8 & 4 & 2.5 & \\
\hline Very high & 17 & 7.3 & 8 & 10.4 & 9 & 5.7 & & 2 & .8 & 1 & 1 & 1 & .6 & \\
\hline Cigarettes per day & & & & & & & .3 & & & & & & & .05 \\
\hline $1-5$ & 53 & 22.5 & 17 & 21.8 & 36 & 22.8 & & 53 & 33.8 & 12 & 22.2 & 41 & 39.8 & \\
\hline $6-10$ & 73 & 30.9 & 18 & 23.1 & 55 & 34.8 & & 46 & 23.3 & 21 & 38.9 & 25 & 24.3 & \\
\hline $11-15$ & 44 & 18.6 & 18 & 23.1 & 26 & 16.5 & & 25 & 15.9 & 12 & 22.2 & 13 & 12.6 & \\
\hline $16-20$ & 48 & 20.3 & 18 & 23.1 & 30 & 19.0 & & 28 & 17.8 & 7 & 13.0 & 21 & 20.4 & \\
\hline $21-30$ & 15 & 6.4 & 5 & 6.4 & 10 & 6.3 & & 5 & 3.2 & 2 & 3.7 & 3 & 2.9 & \\
\hline $31+$ & 3 & 1.3 & 2 & 2.6 & 1 & .6 & & 0 & 0 & 0 & 0 & 3 & 0 & \\
\hline History of cigarette smoking & & & & & & & 6 & & & & & & & .4 \\
\hline Less than 6 mo & 8 & 3.4 & 1 & 1.3 & 7 & 4.4 & & 9 & 5.7 & 2 & 3.7 & 7 & 6.8 & \\
\hline $6-12 \mathrm{mo}$ & 20 & 8.5 & 6 & 7.7 & 14 & 8.9 & & 6 & 3.8 & 4 & 7.4 & 2 & 1.9 & \\
\hline $1-5$ years & 74 & 31.4 & 23 & 29.5 & 51 & 32.3 & & 55 & 35.3 & 18 & 33.3 & 37 & 35.9 & \\
\hline 5 years or greater & 134 & 56.8 & 48 & 61.5 & 86 & 54.4 & & 87 & 55.4 & 30 & 55.6 & 57 & 55.3 & \\
\hline
\end{tabular}

* “Total” pertains to the total sample. ${ }^{1} \mathrm{SC}$ : standard care. ${ }^{2} \mathrm{EI}$ : enhanced intervention. ${ }^{3} \mathrm{P}$ value reflects significance of comparisons between conditions (SC versus EI). No final assessment data were gathered for depressive symptoms (CES-D) and stages of change for cessation (dashes indicate no data). Percentages for each variable may not add up to 100 because of missing data. 


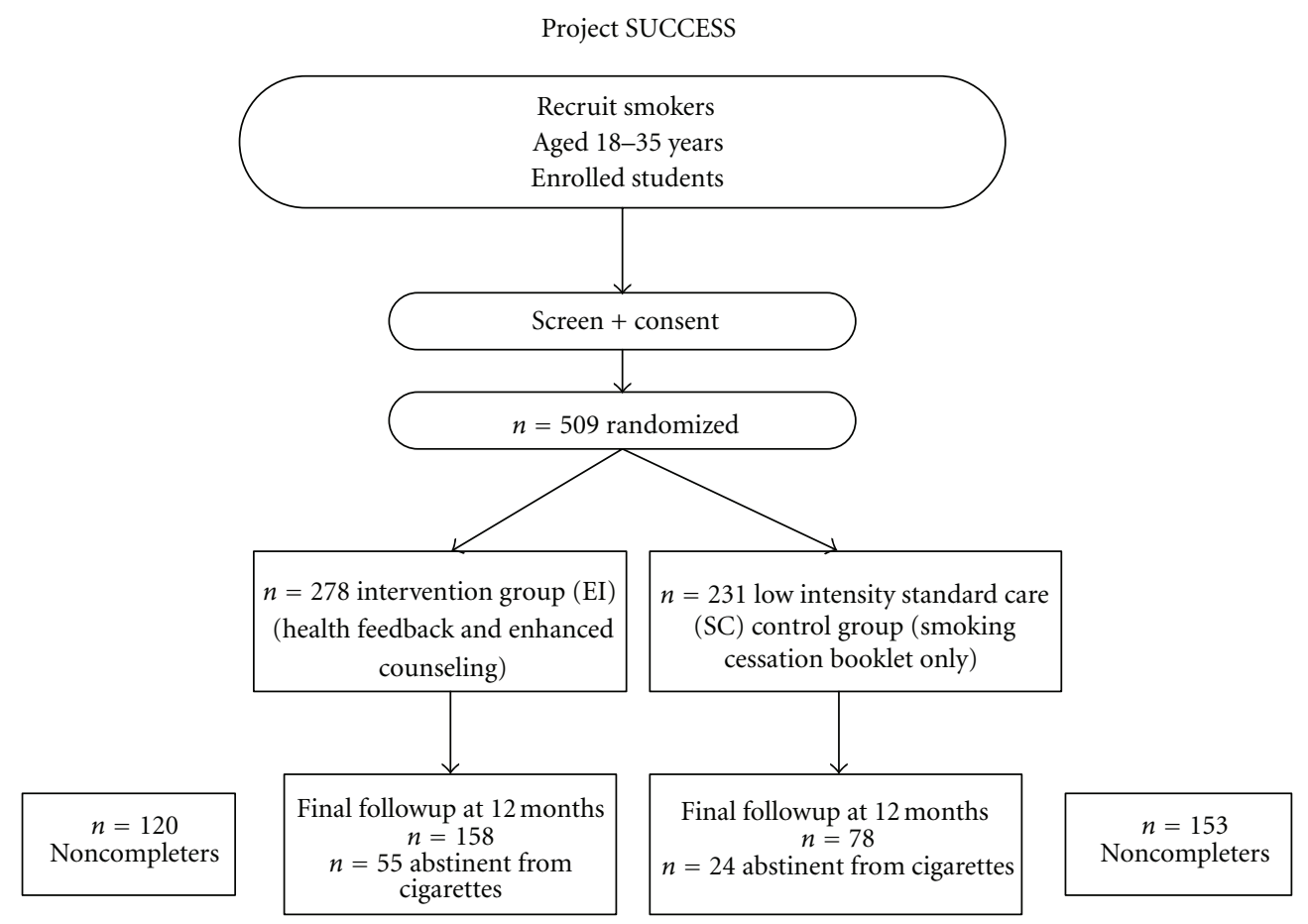

FIgURE 1

EI group than SC. Cessation rates reported from Project SUCCESS from both groups are higher than the $8.2 \%$ for 18 to 24-year-olds in the National Health Interview Survey [31].

No differences were detected between participant characteristics for noncompleters and those who were retained. At no time did participants indicate they dropped because of dissatisfaction with the program. Attrition from smoking cessation trials and clinical trials in general is frequent and a challenge for researchers [32]. For Project SUCCESS, when asked about reasons for dropping, the majority of participants cited they were too busy and had time conflicts. For the EI participants, perhaps attending the 30- to 60-minute counseling sessions was considered burdensome. Another reason for attrition could have been related to participants feeling uncomfortable when discussing ambivalence about making smoking cessation attempts. If participants decided that they were not going to make a quit attempt, participants might have left the study without explanation to avoid embarrassment when informing the counselor. Participants in the control group may have decided to leave the study because being in the control group was perceived to be of limited benefit. The results of screening for depression and alcohol use provided some indication that addressing underlying depression and problematic alcohol use may be a possibility for fortifying future interventions. Another smoking cessation study reported using two methods for retaining participants-reminders on the refrigerator and a telephone call from the study principal investigator [33]. Neither method was effective in retaining participants. More research is needed in optimizing participant retention.

The program was designed to facilitate interested smokers in the EI group to advance in the stages of change toward cessation. It is reasonable to assume that motivation for smoking cessation fades over time, the frequency of relapse increases, and this diverts attention from cessation. For those interested in starting a smoking cessation program on the college campus, the greatest expenses to anticipate will be personnel costs. The salaries for the smoking cessation counselors will depend on criteria set for counselor preparation and credentials.

Notwithstanding behavioral interventions, individuals in smoke-free environments are more likely to quit. Conversely, Project SUCCESS was conducted on a campus where smoking outdoors is allowed and cigarettes were sold on campus. One way to facilitate the successful adoption of tobaccofree policy on campus is to offer on-site smoking cessation services [34]. Project SUCCESS is a step toward achieving the goal of having effective and sustainable smoking cessation programs on campus.

Project SUCCESS has contributed to the research gap regarding smoking cessation among college students. The method for participant recruitment of 509 eligible daily smokers who attended at least one session of the study was a strength as well as the randomized study design. Greater retention was found for those engaged in the active study condition, indicating that personal health feedback was relevant for some individuals and interaction with study staff assisted with retention. The recruitment methods described in this study may help future researchers. The greatest weakness of the study pertained to attrition which can reduce power to detect intervention effectiveness. Misclassification bias was another concern since the analysis was based on a portion of participants' self-report of cessation and may not be accurate. We have focused attention on 
incorporating modules for alleviating depressive symptoms and managing problematic alcohol use into our web-based smoking cessation interventions for college students. We are actively encouraging tobacco-free policies on campus, and joining forces with other groups interested in college health. A planned intervention component includes integrating regular physical activity into cessation programs. A window of opportunity exists for delivering smoking cessation content on social networking sites and by mobile devices, attracting participants and increasing retention among young populations.

\section{Conflict of Interests}

Authors of the paper do not have a direct financial relation with NicAlert or any other study-related commercial interests.

\section{Acknowledgments}

The funding source for Project SUCCESS (Students Using Computerized Coaching to End Smoking Successfully) results from a randomized, controlled trial was NIH/NCI R01 CA69425. A. Prokhorov, M.D., Ph.D., Professor and Director, Tobacco Outreach Education Program Director, eHealth Technology Program, Department of Behavioral Science at the University of Texas MD Anderson Cancer Center, was the Principal Investigator. He is the corresponding author for this paper.

\section{References}

[1] "CDC grand rounds: current opportunities in tobacco control," Morbidity and Mortality Weekly Report, vol. 59, no. 16, pp. 487-492, 2010.

[2] Office of Surgeon General, Prevention of Tobacco Use Among Youth and Young Adults, Department of Health and Human Services, 2012.

[3] P. M. Ling, T. B. Neilands, and S. A. Glantz, "Young adult smoking behavior: a national survey," American Journal of Preventive Medicine, vol. 36, no. 5, pp. 389.e2-394.e2, 2009.

[4] R. Peto, S. Darby, H. Deo, P. Silcocks, E. Whitley, and R. Doll, "Smoking, smoking cessation, and lung cancer in the UK since 1950: combination of national statistics with two case-control studies," British Medical Journal, vol. 321, no. 7257, pp. 323$329,2000$.

[5] H. E. Hutton, L. M. Wilson, B. J. Apelberg et al., "A systematic review of randomized controlled trials: web-based interventions for smoking cessation among adolescents, college students, and adults," Nicotine and Tobacco Research, vol. 13, no. 4, pp. 227-238, 2011.

[6] K. S. Freedman, N. M. Nelson, and L. L. Feldman, "Smoking initiation among young adults in the United States and Canada, 1998-2010: a systematic review," Preventing Chronic Disease, vol. 9, p. E05, 2012.

[7] L. C. Abroms, R. Windsor, and B. Simons-Morton, "Getting young adults to quit smoking: a formative evaluation of the $\mathrm{X}$ Pack program," Nicotine and Tobacco Research, vol. 10, no. 1, pp. 27-33, 2008.

[8] J. A. Cranford, D. Eisenberg, and A. M. Serras, "Substance use behaviors, mental health problems, and use of mental health services in a probability sample of college students," Addictive Behaviors, vol. 34, no. 2, pp. 134-145, 2009.

[9] V. N. Simmons and T. H. Brandon, "Secondary smoking prevention in a university setting: a randomized comparison of an experiential, theory-based intervention and a standard didactic intervention for increasing cessation motivation," Health Psychology, vol. 26, no. 3, pp. 268-277, 2007.

[10] J. L. Obermayer, W. T. Riley, O. Asif, and J. Jean-Mary, "College smoking-cessation using cell phone text messaging," Journal of American College Health, vol. 53, no. 2, pp. 71-78, 2004.

[11] C. Escoffery, L. McCormick, and K. Bateman, "Development and process evaluation of a web-based smoking cessation program for college smokers: innovative tool for education," Patient Education and Counseling, vol. 53, no. 2, pp. 217-225, 2004.

[12] L. C. An, C. Klatt, C. L. Perry et al., "The RealU online cessation intervention for college smokers: a randomized controlled trial," Preventive Medicine, vol. 47, no. 2, pp. 194-199, 2008.

[13] R. Wellman, R. McMillen, and J. DiFranza, "Assessing college students' autonomy over smoking with the hooked on nicotine checklist," Journal of American College Health, vol. 56, no. 5, pp. 549-553, 2008.

[14] R. Murphy-Hoefer, R. Griffith, L. L. Pederson, L. Crossett, S. R. Iyer, and M. D. Hiller, "A review of interventions to reduce tobacco use in colleges and universities," American Journal of Preventive Medicine, vol. 28, no. 2, pp. 188-200, 2005.

[15] N. J. Montalto and W. O. Wells, "Validation of self-reported smoking status using saliva cotinine: a rapid semiquantitative dipstick method," Cancer Epidemiology Biomarkers and Prevention, vol. 16, no. 9, pp. 1858-1862, 2007.

[16] A. V. Prokhorov, C. Rosenblum, R. T. Fouladi et al., "Computer-assisted, counselor-delivered smoking cessation counseling for community college students: intervention approach and sample characteristics," Journal of Child \& Adolescent Substance Abuse, vol. 16, no. 3, pp. 35-62, 2007.

[17] A. V. Prokhorov, T. Yost, M. Mullin-Jones et al., "'Look At Your Health': outcomes associated with a computer-assisted smoking cessation counseling intervention for community college students," Addictive Behaviors, vol. 33, no. 6, pp. 757771, 2008.

[18] J. O. Prochaska, C. C. DiClemente, and J. C. Norcross, "In search of how people change: applications to addictive behaviors," American Psychologist, vol. 47, no. 9, pp. 1102-1114, 1992.

[19] W. R. Miller and S. Rollnick, Motivational Interviewing: Preparing People For Change, Guilford Press, New York, NY, USA, 2nd edition, 2002.

[20] I. Berlin, A. Radzius, J. E. Henningfield, and E. T. Moolchan, "Correlates of expired air carbon monoxide: effect of ethnicity and relationship with saliva cotinine and nicotine," Nicotine and Tobacco Research, vol. 3, no. 4, pp. 325-331, 2001.

[21] J. F. Morris and W. Temple, "Spirometric "lung age" estimation for motivating smoking cessation," Preventive Medicine, vol. 14, no. 5, pp. 655-662, 1985.

[22] G. W. Comstock, W. J. Brownlow, R. W. Stone, and P. E. Sartwell, "Cigarette smoking and changes in respiratory findings," Archives of Environmental Health, vol. 21, no. 1, pp. 50-57, 1970.

[23] G. W. Comstock, M. S. Tockman, K. J. Helsing, and K. M. Hennesy, "Standardized respiratory questionnaires: comparison of the old with the new," American Review of Respiratory Disease, vol. 119, no. 1, pp. 45-53, 1979. 
[24] G. Baldwin and G. D. Shean, "A taxometric study of the center for epidemiological studies depression scale," Genetic, Social, and General Psychology Monographs, vol. 132, no. 2, pp. 101128, 2006.

[25] C. C. DiClemente, J. O. Prochaska, S. K. Fairhurst, W. F. Velicer, M. M. Velasquez, and J. S. Rossi, "The process of smoking cessation: an analysis of precontemplation, contemplation, and preparation stages of change," Journal of Consulting and Clinical Psychology, vol. 59, no. 2, pp. 295-304, 1991.

[26] W. F. Velicer, C. C. DiClemente, J. O. Prochaska, and N. Brandenburg, "Decisional balance measure for assessing and predicting smoking status," Journal of Personality and Social Psychology, vol. 48, no. 5, pp. 1279-1289, 1985.

[27] J. R. Hughes and D. Hatsukami, "Signs and symptoms of tobacco withdrawal," Archives of General Psychiatry, vol. 43, no. 3, pp. 289-294, 1986.

[28] W. F. Velicer, C. C. Diclemente, J. S. Rossi, and J. O. Prochaska, "Relapse situations and self-efficacy: an integrative model," Addictive Behaviors, vol. 15, no. 3, pp. 271-283, 1990.

[29] T. F. Heatherton, L. T. Kozlowski, R. C. Frecker, and K. O. Fagerström, "The Fagerstrom test for nicotine dependence: a revision of the fagerstrom tolerance questionnaire," British Journal of Addiction, vol. 86, no. 9, pp. 1119-1127, 1991.

[30] K. S. Calabro and A. V. Prokhorov, "Respiratory symptoms after smoking cessation among college students," Pediatric, Allergy, Immunology, and Pulmonology, vol. 24, no. 4, pp. 215219, 2011.

[31] Centers for Disease Control, "Quitting smoking among adults-United States, 2001-2010," Morbidity and Mortality Weekly Report, vol. 60, no. 44, 2011.

[32] D. Lindström, I. Sundberg-Petersson, J. Adami, and H. Tönnesen, "Disappointment and drop-out rate after being allocated to control group in a smoking cessation trial," Contemporary Clinical Trials, vol. 31, no. 1, pp. 22-26, 2010.

[33] E. Severi, C. Free, R. Knight, S. Robertson, P. Edwards, and E. Hoile, "Two controlled trials to increase participant retention in a randomized controlled trial of mobile phonebased smoking cessation support in the United Kingdom," Clinical Trials, vol. 8, no. 5, pp. 654-660, 2011.

[34] S. Gajendra, D. J. Ossip, R. J. Panzer, and S. McIntosh, "Implementing a smoke-free campus: a medical center initiative," Journal of Community Health, vol. 36, no. 4, pp. 684-692, 2011. 


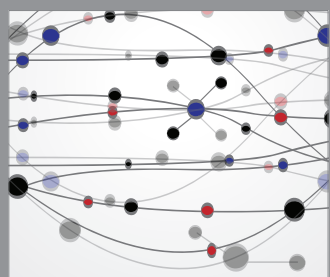

The Scientific World Journal
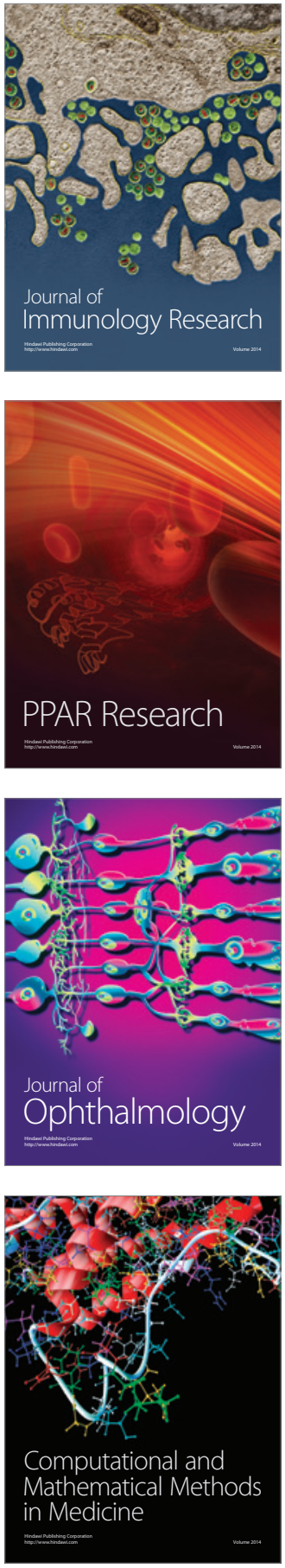

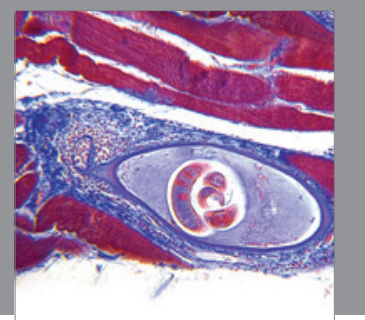

Gastroenterology

Research and Practice
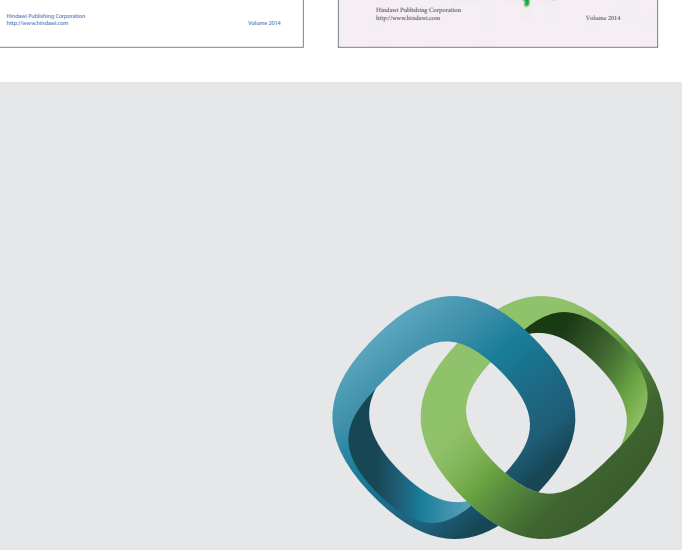

\section{Hindawi}

Submit your manuscripts at

http://www.hindawi.com
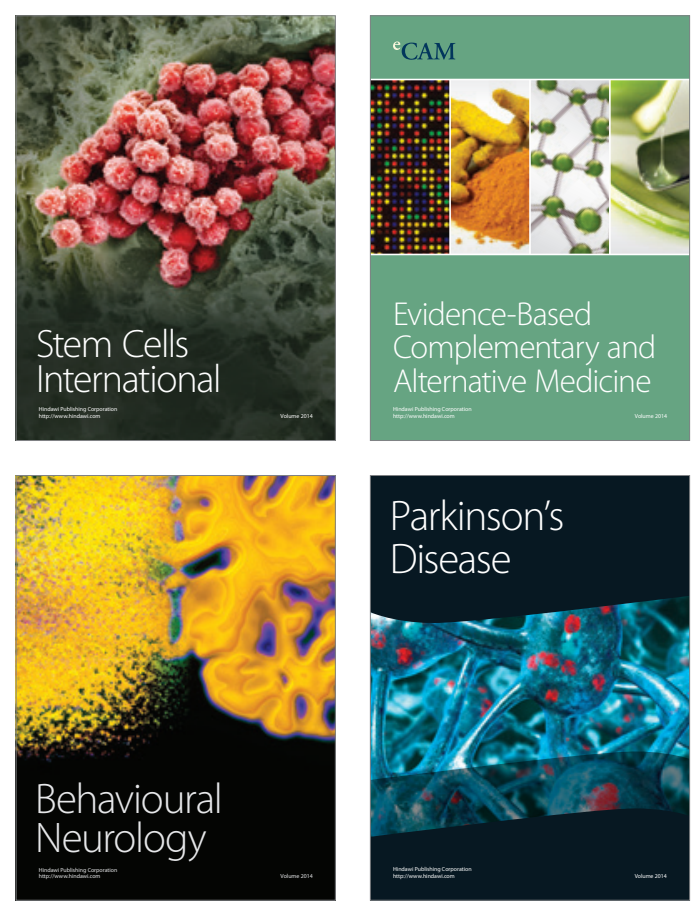

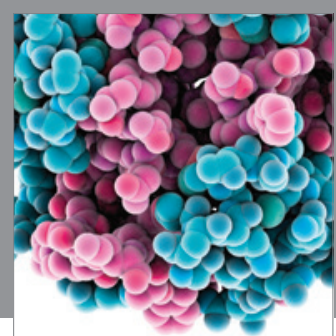

Journal of
Diabetes Research

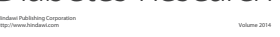

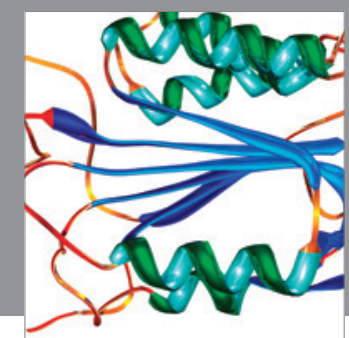

Disease Markers
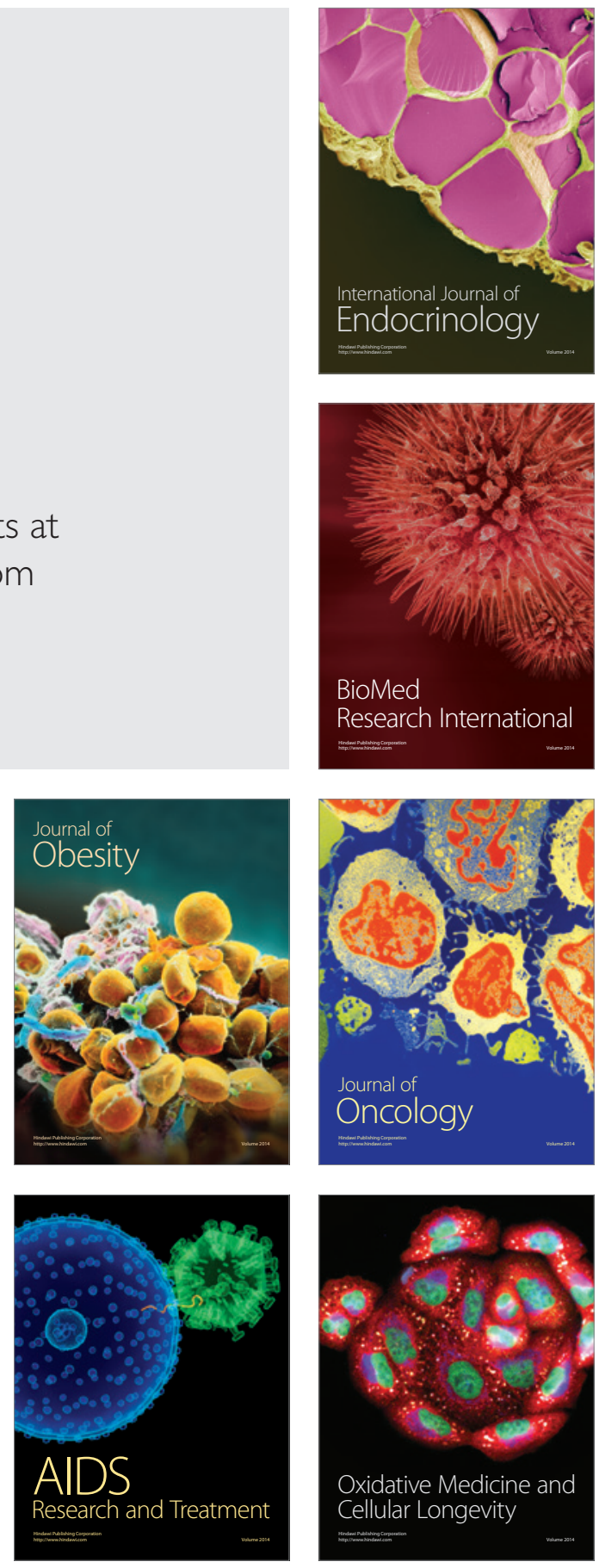